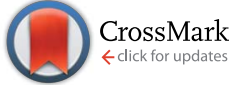

Cite this: RSC Adv., 2017, 7, 16637

\title{
The effect of oxygen on the microwave-assisted synthesis of carbon quantum dots from polyethylene glycol $\uparrow$
}

\begin{abstract}
Yunyang Zhao, Songlin Zuo* and Meng Miao
Fluorescent carbon quantum dots (CQDs) were synthesized using polyethylene glycol (PEG) as both the carbon source and solvent in various atmospheres including air, nitrogen, carbon dioxide and oxygen by following a microwave irradiation method. The CQDs were characterized by performing ultravioletvisible absorption spectroscopy, Fourier transform infrared spectroscopy, transmission electron microscopy, and fluorescence spectroscopy. We found that different reaction atmospheres yielded significantly different reaction rates and properties of the CQDs. In particular, including oxygen in the synthesis of the CQDs clearly shortened the reaction time, significantly enhanced the photoluminescence (PL) intensity and quantum yield (PLQY) of the CQDs, and extended their average lifetime. The PLQY and fluorescence lifetime of the CQDs were improved from $5.78 \%$ and $2.4 \mathrm{~ns}$, respectively, when synthesized in a medium open to air to $7.84 \%$ and $3.0 \mathrm{~ns}$, respectively, when synthesized in an $\mathrm{O}_{2}$ atmosphere. The CQDs synthesized using microwave irradiation showed strong blue fluorescence under ultraviolet light.
\end{abstract}

Received 13th February 2017 Accepted 4th March 2017

DOI: $10.1039 / \mathrm{c} 7 \mathrm{ra01804e}$

rsc.li/rsc-advances
Bottom-up approaches have received more attention for preparing CQDs by dehydration, carbonization and aromatization of carbonaceous precursors (L-ascorbic acid, ${ }^{17}$ glucose ${ }^{18}$ and citric acids ${ }^{5}$ ). However, these CQDs typically require further surface oxidation and/or passivation to render or enhance their fluorescence and simultaneously the hydrophilicity of their surfaces. ${ }^{19}$ Thus, it is still a challenge to develop a simple method for synthesizing self-passivated CQDs.

Polyethylene glycol (PEG) is a polyhydroxy compound and has been used as a surface passivating agent in CQD syntheses. Recently, PEG has attracted much interest as a carbonaceous precursor in the synthesis of CQDs due to its non-toxicity, nonimmunogenicity and reactivity with other biomolecules. ${ }^{20-22}$ So far, a few reports on the synthesis of fluorescent CQDs from PEG have appeared. PEG, as a water-soluble oligomer, shows excellent potential in improving the solubility and biocompatibility of nanoparticles. ${ }^{23}$ In 2013, Rui-Jun Fan et al. ${ }^{24}$ obtained CQDs derived from an aqueous solution of PEG 400 by carrying out a hydrothermal treatment of this solution at $120{ }^{\circ} \mathrm{C}$. However, the photoluminescence quantum yield (PLQY) was very low, only $3.5 \%$, and such a route involving heat operation and intricate post-processes is time-consuming. In 2014, Chen et al. ${ }^{25}$ reported a simple one-pot thermal treatment to fabricate water-soluble fluorescent CQDs from PEG at a high temperature of $160{ }^{\circ} \mathrm{C}$. Recently, Kumar et al. ${ }^{26}$ reported that blue fluorescent CQDs could be directly prepared from PEG 400 by carrying out ultrasonic treatments at various temperatures. Although the CQDs prepared using this method showed a high PLQY, this approach requires a long ultrasonic treatment time of $2.5 \mathrm{~h}$.
College of Chemical Engineering, Jiangsu Key Laboratory of Biomass-Based Green Fuels and Chemicals, Nanjing Forestry University, Nanjing 210037, China. E-mail: zslnl@hotmail.com; zslnl@njfu.com.cn; Fax: +86 25 85418873; Tel: +86 2585428840 $\dagger$ Electronic supplementary information (ESI) available. See DOI: 10.1039/c7ra01804e 
Microwave irradiation, by interacting with the matter in a solution, can elevate the temperature of the solution up to $1000{ }^{\circ} \mathrm{C}$ within only one minute, ${ }^{27}$ and use of such irradiation is thus energy efficient. Some efforts have made to synthesize CQDs by microwave heating of aqueous solutions of PEG or PEG and sugar in various conditions. ${ }^{28,29}$ Nevertheless, little attention was paid to the effect of the atmosphere on the formation of CQDs from PEG in these liquid reaction processes. In our current work, we discovered that oxygen could be used to accelerate the synthesis of CQDs from PEG and remarkably influenced the properties of the CQDs using PEG as a singlecomponent precursor without subsequent passivation.

\section{Experimental}

\section{Synthesis of CQDs}

PEG with a molecular weight of 400 Da (PEG 400) was purchased from Chengdu Kelong Chemical Reagent Factory (Cheng Du, China), and was used as both a carbon source and solvent. For synthesizing CQDs, a volume of $40 \mathrm{~mL}$ of PEG 400 was transferred into a dried $100 \mathrm{~mL}$ single-necked flask and then heated in a $500 \mathrm{~W}$ microwave reactor (MAS-I, Sineo Microwave Chemistry Technology Co., Ltd, Shanghai, China) to $100{ }^{\circ} \mathrm{C}$ with a stirring speed of $500 \mathrm{rpm}$ by using a magnetic agitator. During the synthesis, a gas $\left(\mathrm{O}_{2}, \mathrm{~N}_{2}\right.$ or $\left.\mathrm{CO}_{2}\right)$ was bubbled into the flask, or the flask was left open to air, in order to investigate the effects of these atmospheres on the synthesized CQDs. Finally, in each case, a golden yellow solution containing CQDs was obtained and named "CQDs- $a-b, Q(A)=c \mathrm{~mL} \min ^{-1}$ ", where $a, b, A$ and $c$ indicate the reaction time, the reaction temperature, the name of atmosphere involving the PEG, and the flow rate of the corresponding atmosphere, respectively. For example, "CQDs-60$100, Q\left(\mathrm{O}_{2}\right)=100 \mathrm{~mL} \mathrm{~min}{ }^{-1}$ " stands for CQDs prepared from $40 \mathrm{~mL}$ PEG 400 by carrying out a $500 \mathrm{~W}$ microwave heating at $100{ }^{\circ} \mathrm{C}$ for $60 \mathrm{~min}$ and involving $100 \mathrm{~mL} \mathrm{~min}^{-1}$ oxygen in the reaction. When CQDs were synthesized under an open-air atmosphere, $Q(A)$ was omitted.

\section{Characterizations}

The fluorescence of the CQDs was measured by using an LS 55 fluorescence spectrophotometer (PE Corporation, USA). The CQD dimensions were determined by using a high-resolution transmission electron microscope (HRTEM, JEOL, Japan) operating at an accelerating voltage of $200 \mathrm{kV}$. The samples for the TEM were prepared by drying $5 \mu \mathrm{L}$ of an isopropanol solution of PEG containing CQDs on a carbon-coated copper grid (thinner pure carbon support films of $7-10 \mathrm{~nm}$ ) in a vacuum drying oven at $80^{\circ} \mathrm{C}$ for more than three days. Ultraviolet-visible (UV-vis) absorption spectra were obtained using a UV-2450 UVvis spectrophotometer (Shimadzu Corporation, Japan). Fourier transform infrared (FTIR) spectra were acquired from a 360 FTIR spectrometer (Thermo Nicolet Corporation, USA) with wavenumbers ranging from 500 to $4000 \mathrm{~cm}^{-1}$. Time-resolved fluorescence intensity decay of the CQDs was recorded using a FM-4P-TCSPC spectrofluorometer (Horiba Jobin Yvon, France) with a laser excitation wavelength of $375 \mathrm{~nm} .{ }^{1} \mathrm{H}$ NMR and ${ }^{13} \mathrm{C}$
NMR spectra of the CQDs and raw PEG were recorded in deuterated chloroform on an AVANCE III HD (Bruker Biospin, Switzerland) $600 \mathrm{MHz}$ spectrometer.

$$
\mathrm{PLQY}=\frac{A(\mathrm{em})_{\mathrm{s}}-A(\mathrm{em})_{\mathrm{b}}}{A(\mathrm{sc})_{\mathrm{b}}-A(\mathrm{sc})_{\mathrm{s}}}
$$

\section{Quantum yield and fluorescence lifetime measurements}

In this work, an FM-4P-TCSPC transient fluorescence spectrometer was used to directly measure the absolute PLQY and fluorescence lifetime of the CQDs. The absolute PLQY of the CQDs was calculated using the formula. where $A(\mathrm{em})$ and $A(\mathrm{sc})$ are the integrated fluorescence emission intensity and integrated Rayleigh scattering intensity, respectively, and the subscripts s and b refer to sample (CQDs) and blank (solvent), respectively.

Fluorescence lifetime, defined as the characteristic period that the CQDs remained in their excited states prior to returning to their ground states, was determined by using a transient fluorescence spectrometer with a $370 \mathrm{~nm}$-wavelength laser. The decay trace of the PL intensity of the CQDs was fitted using multi-exponential functions $I(t)$ based on a non-linear least squares analysis using the equation:

$$
I(t)=\sum_{i}\left[\alpha_{i} \exp \left(\frac{-t}{\tau_{i}}\right)\right]
$$

where $\alpha_{i}$ are the fractional contributions of the time-resolved decay lifetime of $\tau_{i}$.

\section{Results and discussion}

\section{Effect of atmosphere on CQD synthesis}

Fig. 1(A) shows fluorescence emission spectra and corresponding optical images (inset) of CQDs synthesized in different atmospheres. They consistently showed CQDs synthesized in pure oxygen and in air to have much stronger PL intensity levels than those in $\mathrm{N}_{2}$ and in $\mathrm{CO}_{2}$, with the strongest PL intensity for pure oxygen. Fig. 1(B) shows UV-vis absorption spectra of the CQDs. The CQDs synthesized in pure oxygen clearly yielded the broadest and strongest absorption band in the UV-visible region with wavelengths from $225 \mathrm{~nm}$ to $325 \mathrm{~nm}$,
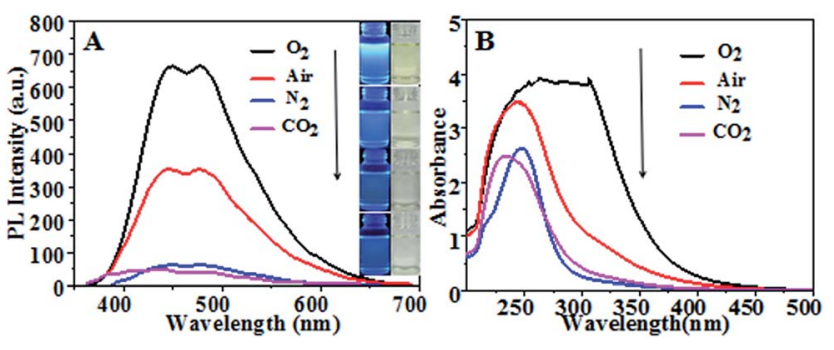

Fig. 1 (A) Fluorescence emission spectra of the various CQDs. (Insets are optical images of CQDs synthesized in various gas atmospheres under $365 \mathrm{~nm}$-wavelength UV light (left) and sunlight (right)). (B) UV-vis absorption spectra of CQDs synthesized at $100{ }^{\circ} \mathrm{C}$ for $60 \mathrm{~min}$ in different gas atmospheres with a flow rate of $100 \mathrm{~mL} \mathrm{~min}^{-1}$. 
indicating these CQDs to have the greatest relative number of aromatic $\mathrm{sp}^{2}$ centers. ${ }^{30-32}$ The CQDs synthesized in the inert $\mathrm{N}_{2}$ and $\mathrm{CO}_{2}$ atmospheres exhibited relatively low PL intensities and weak UV adsorption.

Fig. 2 shows TEM images of the synthesized CQDs and their corresponding particle size distributions. The CQDs in Fig. 2(A), (C) and (D) were observed to be very well dispersed and to have a narrow size distribution, while those in Fig. 2(B), i.e., "CQDs60-100, in air", were poorly dispersed with a relatively broad size distribution. In addition, the "CQDs-60-100, in air" sample was observed to have a rougher surface and larger mean particle size than the other three samples, possibly resulting in the low PLQYs of these CQDs, which are shown in Table 1. To test the effect of the CQDs synthesis conditions on the CQDs fluorescence, the CQDs solutions, all at the same concentration, were excited with the same number of photons. More photons were observed to be emitted from the fluorescent CQDs having the uniform particle size distribution and good dispersibility than from the other CQDs. Moreover, the fluorescence emission of the CQDs having the broad particle size distribution with poor dispersibility was more self absorbed than the fluorescence emission of the other CQDs since the former CQDs cast a greater "shade".

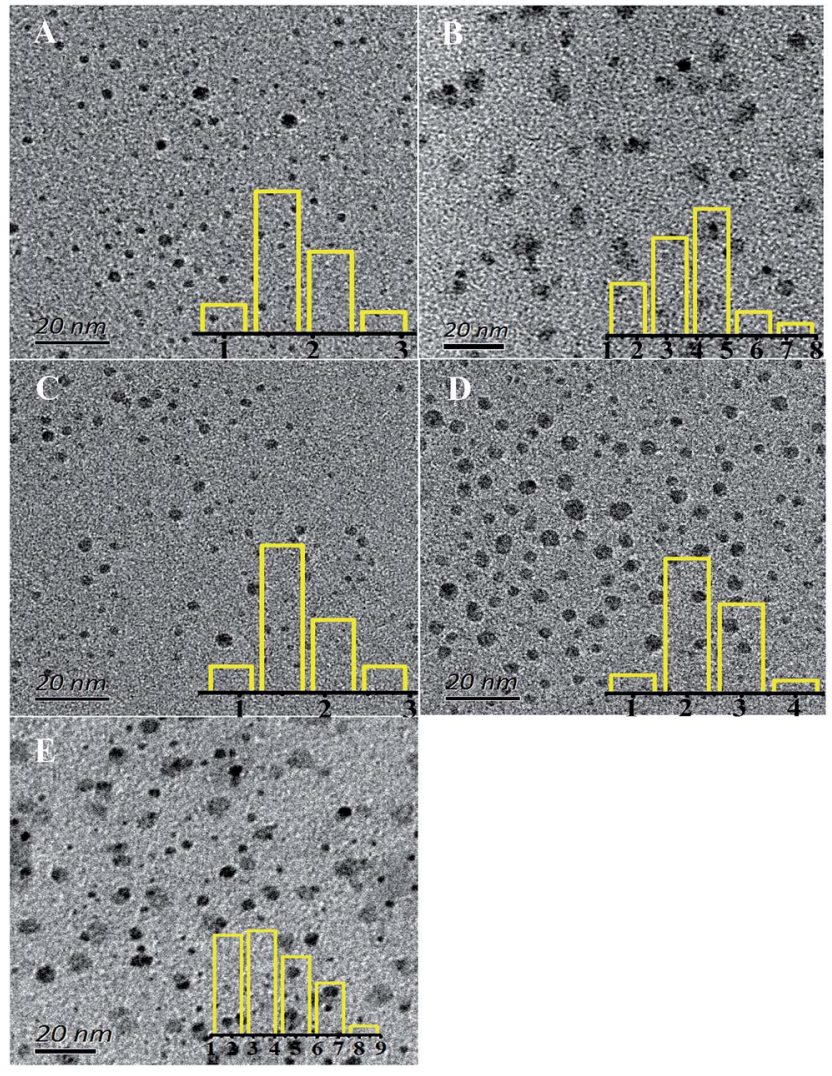

Fig. 2 TEM images of the synthesized CQDs with different reaction

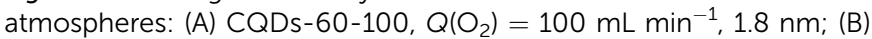
CQDs-60-100, air, $3.8 \mathrm{~nm}$; (C) CQDs-60-100, $Q\left(\mathrm{~N}_{2}\right)=100 \mathrm{~mL} \mathrm{~min}{ }^{-1}$ $1.7 \mathrm{~nm}$; (D) CQDs-60-100, $Q\left(\mathrm{CO}_{2}\right)=100 \mathrm{~mL} \mathrm{~min}^{-1}, 2.4 \mathrm{~nm}$. (E) CQDs-

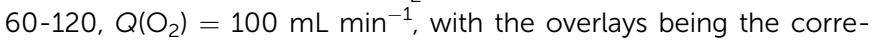
sponding particle size distribution histogram.
Table 1 The PLQY and average lifetime $(t)$ values of the CQDs

\begin{tabular}{|c|c|c|c|}
\hline CQDs samples & PLQY, \% & $\tau, \mathrm{ns}$ & Size, nm \\
\hline CQDs-60-100, in air & 4.05 & 0.33 & 3.8 \\
\hline CQDs-60-100, $Q\left(\mathrm{O}_{2}\right)=100 \mathrm{~mL} \mathrm{\textrm {min } ^ { - 1 }}$ & 5.82 & 0.43 & 1.8 \\
\hline CQDs-60-100, $Q\left(\mathrm{~N}_{2}\right)=100 \mathrm{~mL} \min ^{-1}$ & 4.45 & 0.28 & 1.7 \\
\hline CQDs-60-100, $Q\left(\mathrm{CO}_{2}\right)=100 \mathrm{~mL} \mathrm{~min}^{-1}$ & 8.83 & $<0.1$ & 2.4 \\
\hline
\end{tabular}

Table 1 lists the PLQY and average lifetime values of the synthesized CQDs. The inclusion of an oxygen atmosphere during the synthesis was found to increase both the PL intensity and the PLQY of the CQDs. The enhanced PLQY of the CQDs in the $\mathrm{O}_{2}$ atmosphere was inferred, based on eqn (1-1) in section 2.2, to be due to the improvement of their PL intensity. The significantly enhanced PLQY in $\mathrm{CO}_{2}$ may have been caused by an increased $A(\mathrm{sc})$ (see eqn (1-1)). Using eqn (1-2) and the resulting equation $\tau=\sum \alpha_{i} \tau_{i}$, the average lifetimes of the CQDs were calculated from the fluorescence decay curves presented in Fig. S1. $\dagger$ The results showed that $\mathrm{O}_{2}$ involved in the reaction can also extend the average lifetime of CQDs from $0.33 \mathrm{~ns}$ to $0.43 \mathrm{~ns}$ (Table 1). Both the $\mathrm{N}_{2}$ and $\mathrm{CO}_{2}$ atmospheres shortened the lifetime of the synthesized CQDs. The average lifetime of

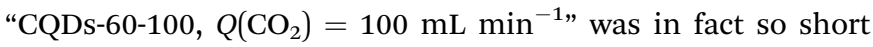
that it could not be determined using our instrument because its fluorescence decay curve (red curve) coincided with that of the silicone background (blue curve) with hardly any differences (in Fig. S1(D)†े).

\section{Effect of reaction conditions in oxygen atmosphere}

Reaction temperature. In order to study the influence of the reaction temperature on the properties of the CQDs, PEG 400 was heated using $500 \mathrm{~W}$ microwave irradiation to temperatures of $90{ }^{\circ} \mathrm{C}, 100^{\circ} \mathrm{C}, 110^{\circ} \mathrm{C}$, or $120^{\circ} \mathrm{C}$ for $60 \mathrm{~min}$. Fig. 3(A) shows the effect of the reaction temperature on the PL intensities of the CQDs. The PL intensities were generally observed to increase and then decrease as the reaction temperature was increased in this range. Note that the excitation wavelength used increased with temperature. Fig. 3(B) shows the UV-vis absorption spectra at the indicated reaction temperatures. The absorption bands became broader and stronger when the reaction temperature was increased from $85{ }^{\circ} \mathrm{C}$ to $120{ }^{\circ} \mathrm{C}$. A very strong absorption appeared at $320 \mathrm{~nm}$ when the temperature was increased to
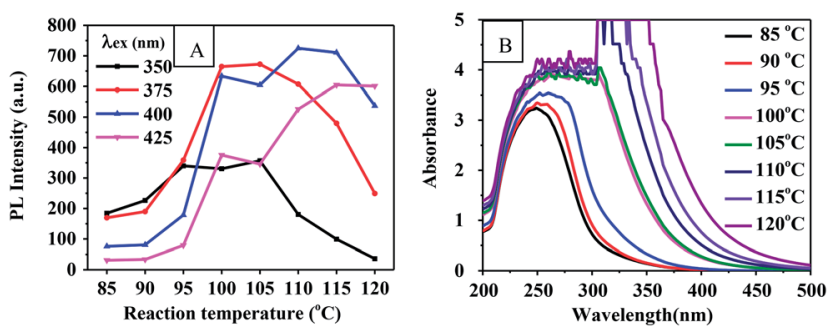

Fig. 3 (A) PL intensity-reaction temperature curve of CQDs prepared for $60 \mathrm{~min}$. (B) UV-vis absorption spectrum of CQDs at corresponding reaction temperatures. 
above $110{ }^{\circ} \mathrm{C}$. However, the absorbance and PL intensity of the CQDs were observed to sharply increase with an increase in the reaction temperature from 95 to $100{ }^{\circ} \mathrm{C}$ and then to slowly increase from 100 to $105{ }^{\circ} \mathrm{C}$. This result may be attributed to the rapid evaporation of the water produced from the reaction at $100{ }^{\circ} \mathrm{C}$ having shifted the reaction equilibrium advantageously through a continuous removal of the product.

Optical images of these CQD solutions are shown in Fig. S2. $\dagger$ These solutions, when obtained at temperatures above $100{ }^{\circ} \mathrm{C}$, were observed to be dark. Although using a higher temperature can shorten the reaction time, it may lead to an uneven size distribution of the CQD particles or excessive carbonization. This supposition was supported by the TEM image of the CQDs synthesized at $120^{\circ} \mathrm{C}$ for $1 \mathrm{~h}$ as shown in Fig. 2(E). The reactions at temperatures below $100{ }^{\circ} \mathrm{C}$ needed to be carried out for a long time and produced CQDs with weak PL intensities. Fig. $\mathrm{S} 3 \dagger$ shows the fluorescence emission spectra of CQDs obtained at various reaction temperatures for $60 \mathrm{~min}$. The PL intensities of the synthesized CQDs increased first and then slightly decreased between excitation wavelengths of 350 and $425 \mathrm{~nm}$ as the reaction temperature was increased from $90{ }^{\circ} \mathrm{C}$ to $120{ }^{\circ} \mathrm{C}$. The PL intensities of the CQDs peaked at an excitation wavelength of $375 \mathrm{~nm}$ when the reaction temperature was $100{ }^{\circ} \mathrm{C}$, which indicated the optimal reaction temperature to be $100{ }^{\circ} \mathrm{C}$. We also found that the CQDs exhibited an excitation-dependent PL behavior (Fig. S3†), as reported previously. ${ }^{24,29,31}$ With an increase in the excitation wavelength from 300 to $500 \mathrm{~nm}$, the emission from the CQDs gradually shifted to a longer wavelength. Similarly, a red shift for the CQD emission occurred as the reaction temperature was increased. This red shift may have been due to the enhanced aromatization at increased reaction temperatures.

Reaction time. As shown in Fig. 4(A) and (B), the PL intensity of CQDs synthesized in an $\mathrm{O}_{2}$ flow increased as the reaction time was extended from $30 \mathrm{~min}$ to $60 \mathrm{~min}$ and then leveled off. This increased PL intensity was more significant for CQDs synthesized in the oxygen atmosphere than for that in air. This observation suggested that oxygen was involved in the CQD formation reaction. Therefore, using an oxygen atmosphere could significantly reduce the reaction time for synthesizing the CQDs with a high PL intensity. Additionally, the PL intensities of the CQDs obtained using the various reaction times peaked
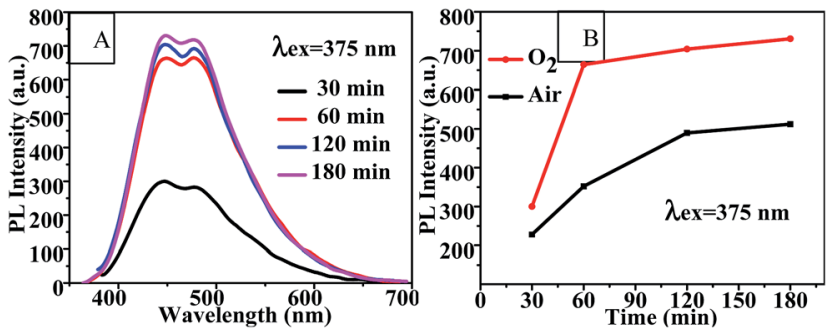

Fig. 4 (A) Fluorescence emission spectra and (B) PL intensity-reaction time curve of CQDs prepared with various different reaction times at $100{ }^{\circ} \mathrm{C}$.
Table 2 The PLQY and average lifetime ( $t$ ) values of CQDs

\begin{tabular}{llll}
\hline CQDs samples & $\lambda_{\text {ex }}$ & PLQY & $\tau$ \\
\hline CQDs-120-100, in air & $375 \mathrm{~nm}$ & $5.78 \%$ & $2.4 \mathrm{~ns}$ \\
CQDs-120-100, $Q\left(\mathrm{O}_{2}\right)=100 \mathrm{~mL} \mathrm{~min}^{-1}$ & $375 \mathrm{~nm}$ & $7.84 \%$ & $3.0 \mathrm{~ns}$
\end{tabular}

at an excitation wavelength of $375 \mathrm{~nm}$, and also exhibited an excitation-dependent PL behavior (Fig. S4 $\dagger$ ).

The PLQY and lifetime values of the CQDs synthesized in air and in oxygen for $120 \mathrm{~min}$ are shown in Table 2 . The lifetimes of these CQDs increased from $0.33 \mathrm{~ns}$ and $0.43 \mathrm{~ns}$, respectively, to $2.4 \mathrm{~ns}$ and $3.0 \mathrm{~ns}$, respectively, (Table 2) as the reaction time was increased from $60 \mathrm{~min}$ (Table 1) to $120 \mathrm{~min}$. And the PLQY increased, simultaneously with PL intensity, from $4.05 \%$ and $5.82 \%$ to $5.78 \%$ and $7.84 \%$ as the reaction time was increased from 60 to $120 \mathrm{~min}$. Moreover, the PLQY and lifetime of the CQDs increased as pure oxygen was bubbled into the reaction solution.

Oxygen flow rate. Fig. 5(A) and (B) show the effects of oxygen flow rate on the fluorescence emission of CQD solutions at an excitation wavelength of $375 \mathrm{~nm}$ and on the PL intensity of the CQDs at various excitation wavelengths. It is clear that the PL intensity of the CQDs could be enhanced by increasing the

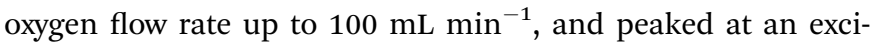
tation wavelength of $375 \mathrm{~nm}$. This result further indicated that oxygen was involved in the synthesis reaction of CQDs. The fluorescence emission spectra of CQDs synthesized with various oxygen flow rates and with a series of excitation wavelengths are shown in Fig. S5.† Similarly, these CQDs also exhibited excitation-dependent PL behavior.

\section{Chemical structure of CQDS}

NMR spectroscopy and FTIR experiments were carried out to determine the chemical structures of the CQDs synthesized under different atmospheres, as shown in Fig. 6 and 7. Slight differences between the ${ }^{1} \mathrm{H}$ NMR spectrum of raw PEG (Fig. 6(A)) and that of the "CQDs-60-100, $Q\left(\mathrm{O}_{2}\right)=100 \mathrm{~mL} \mathrm{~min}^{-1}$ " sample (Fig. 6(E)) were found only in two domains, with chemical shifts of 8-7.5 ppm (assigned to hydrogen of the aromatic ring or $\mathrm{sp}^{2}$ carbon) and 4.5-4.0 ppm (assigned to the protons attached to hydroxyl, ether or carbonyl groups), respectively. ${ }^{33}$ And even less

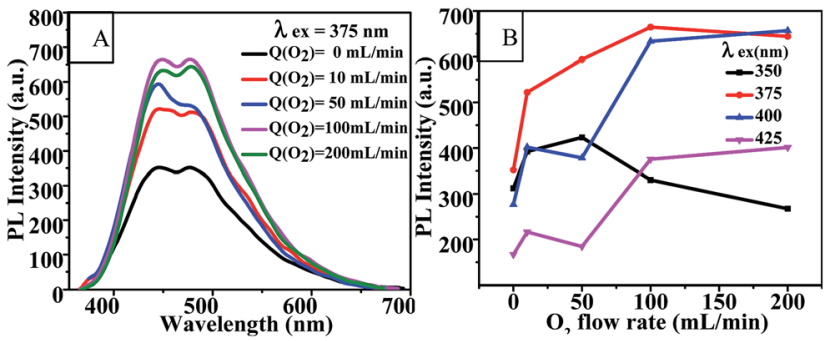

Fig. 5 (A) Fluorescence emission spectra and (B) PL intensity- $\mathrm{O}_{2}$ flow rate curves of CQDs prepared under various oxygen flow rates at $100{ }^{\circ} \mathrm{C}$ for $60 \mathrm{~min}$. $Q\left(\mathrm{O}_{2}\right)=0 \mathrm{~mL} \mathrm{~min}{ }^{-1}$ indicates the CQDs were synthesized in a medium open to air. 

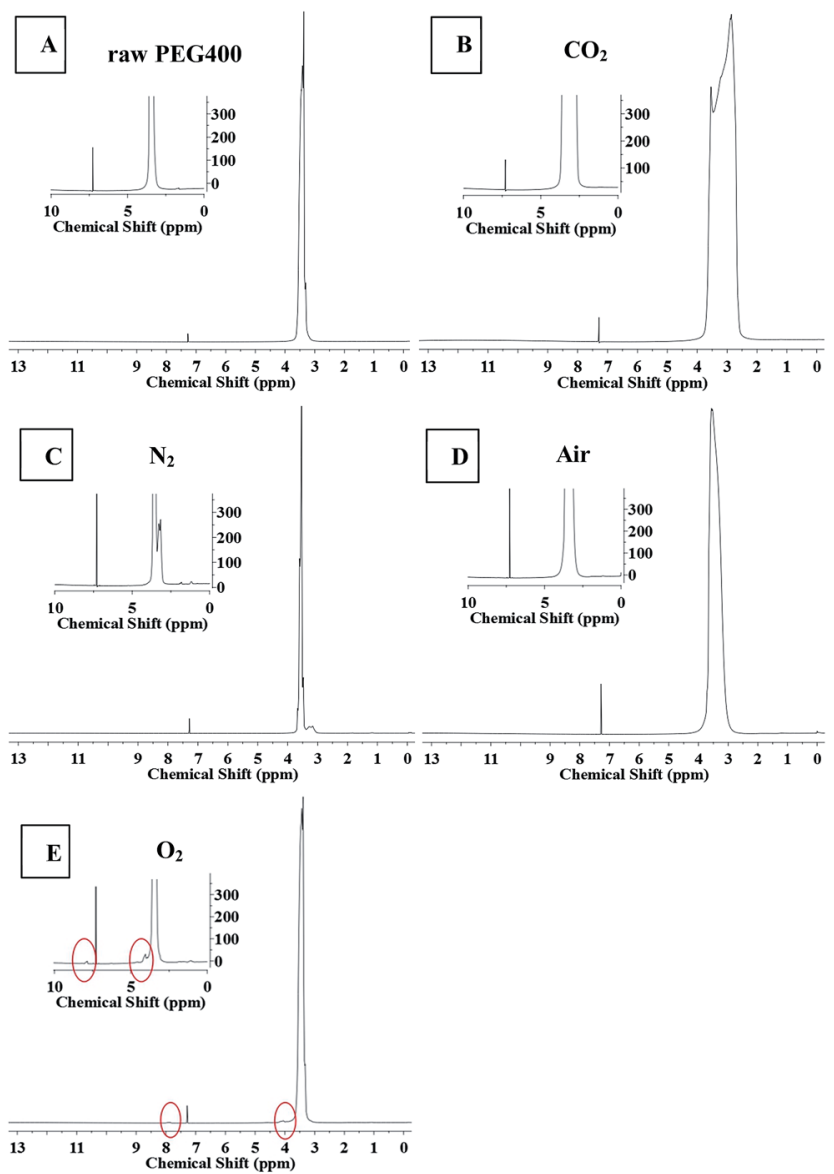

Fig. 6 The ${ }^{1} \mathrm{H}$ NMR spectra of raw PEG and CQDs synthesized in the different atmospheres. (The illustration is a partial enlarged view.) A raw PEG 400, B CO $2, \mathrm{C} \mathrm{N}_{2}, \mathrm{D}$ air, $\mathrm{E} \mathrm{O}_{2}$.

of a difference was found between the ${ }^{1} \mathrm{H}$ NMR spectra of the CQDs synthesized in the other atmospheres. In the ${ }^{1} \mathrm{H}$ NMR spectra of PEG and its derived CQDs, the predominant peak at the chemical shift of $3.5 \mathrm{ppm}$ was attributed to $-\mathrm{CH}_{2}-\mathrm{O}$ (from PEG 400), and a weak peak at about 3 ppm (Fig. 6(B) and (C)) may be assigned to PEG fragments of various molecular weights.

Fig. 7 shows ${ }^{13} \mathrm{C}$ NMR spectra and FTIR spectra. The ${ }^{13} \mathrm{C}$ NMR spectra of the synthesized CQDs were similar and contained four main peaks at chemical shifts of $61.2 \mathrm{ppm}$, $70.2 \mathrm{ppm}$ and $72 \mathrm{ppm}$, and $77.2 \mathrm{ppm}$, which were assigned to carbon atoms in the linkages of $\mathrm{CH}_{2}-\mathrm{O}, \mathrm{CH}_{2}-\mathrm{CH}_{2}$ (from PEG 400), and the deuterated reagent $\mathrm{CDCl}_{3}$, respectively. An obvious difference between the ${ }^{13} \mathrm{C}$ NMR spectra was observed: the CQDs synthesized in an $\mathrm{O}_{2}$ atmosphere yielded a new peak at $160 \mathrm{ppm}$, which was assigned to $\mathrm{C}=\mathrm{C}$ of an aromatic ether or aromatic $\mathrm{sp}^{2}$ carbons. This peak was not observed in the spectra of the CQDs synthesized in the other atmospheres. This result revealed that heating PEG in the oxygen atmosphere produced a higher degree of aromatization than did heating it in inert atmospheres such as nitrogen and carbon dioxide.

The results obtained from FTIR observations (Fig. 7(F)) supported the above-mentioned inference that oxygen promoted the

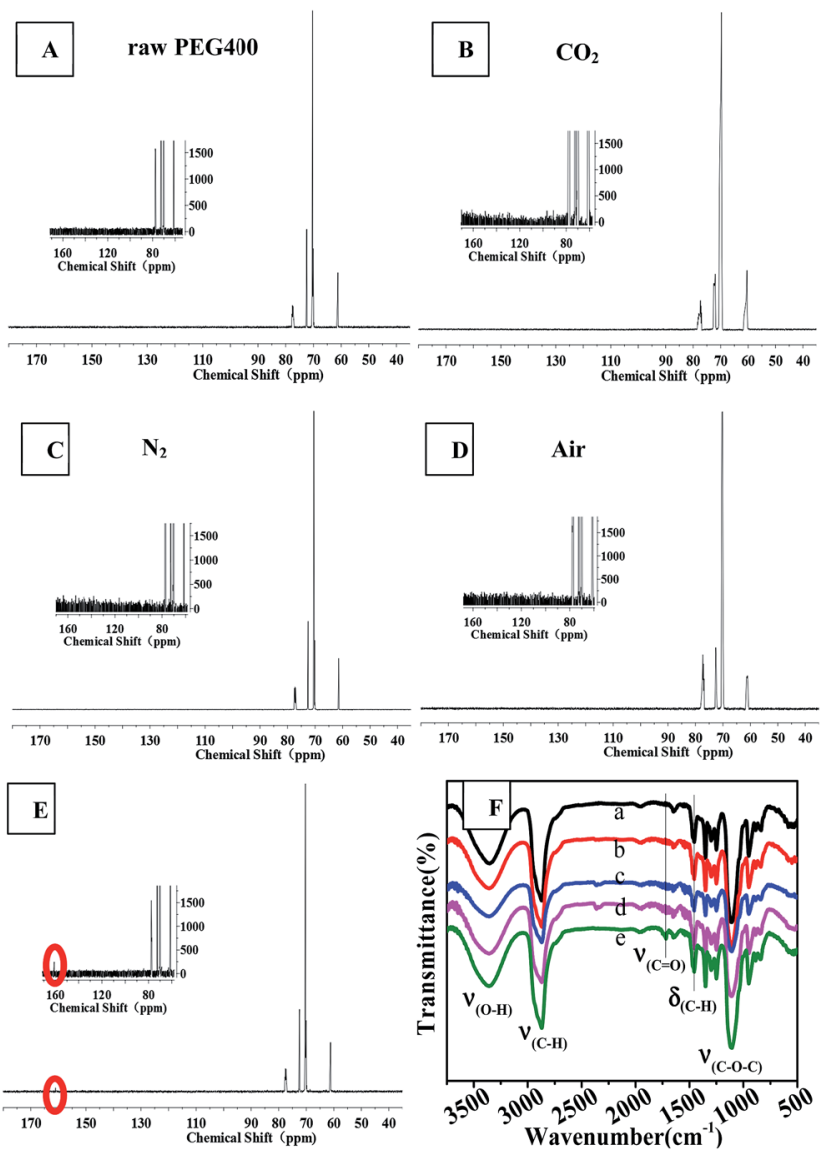

Fig. $7{ }^{13} \mathrm{C}$ NMR (from $A$ to $E$, the illustration is a partial enlarged view) and FTIR (F) spectra of raw PEG and CQDs prepared in different atmospheres: $\mathrm{F}$ (a) raw PEG400, (b) $\mathrm{CO}_{2}$, (c) $\mathrm{N}_{2}$, (d) air, (e) $\mathrm{O}_{2}$.

aromatization of PEG during the synthesis of CQDs. In the FTIR spectra, the bands at $3358 \mathrm{~cm}^{-1}, 2870 \mathrm{~cm}^{-1}$ and $1400-1000 \mathrm{~cm}^{-1}$ (ref. 25) were assigned to the stretching vibrations of $\mathrm{C}-\mathrm{OH}, \mathrm{C}-\mathrm{H}$ and $\mathrm{C}-\mathrm{O}-\mathrm{C}$ linkages, which are characteristic of PEG 400. The synthesized CQDs exhibited many weak peaks in the range of $1500-2000 \mathrm{~cm}^{-1}$, indicative of the formation of aromatic rings due to dehydration. The IR spectrum of "CQDs-60-100, $Q\left(\mathrm{O}_{2}\right)=$

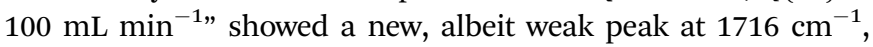
which corresponded to the stretching vibrations of $\mathrm{C}=\mathrm{O}$ in the carboxylic moiety, ${ }^{25}$ and relatively sharp peaks at $1456 \mathrm{~cm}^{-1}$ and $800-900 \mathrm{~cm}^{-1}$, which corresponded to the bending vibration bands of $\mathrm{C}-\mathrm{H}$ and $\mathrm{C}-\mathrm{H}$ bond vibrations in aromatics. ${ }^{11,34,35}$

\section{Mechanism of formation of the CQDs}

Based on the above results, the fluorescence of the CQDs arose from the delocalized $\pi$-electron transition ( $\pi-\pi^{*}$ transition) of the CQDs that absorbed UV-vis light with a wavelength of about $250 \mathrm{~nm}$. In the microwave-assisted formation of CQDs, PEG underwent successive reactions, including dehydrogenation, polymerization, cyclization and aromatization. The general reaction pathway is shown in Scheme 1. The formed CQDs contained oxygen functional groups such as phenolic and alcoholic hydroxyl groups, cyclic ether bonds, and carboxyl 


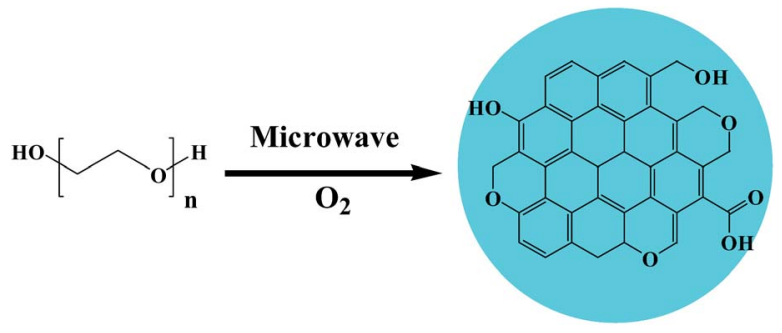

Scheme 1 Microwave-assisted synthesis of CQDs from polyethylene glycol.

groups..$^{25,36-38}$ In proposed specific pathways, ether bonds of PEG first decomposed into free radicals that subsequently isomerized into alcohol hydroxyl groups ${ }^{25,38}$ and these alcohol hydroxyls formed double bonds as a result of an elimination reaction. Here, three conjugated double bonds converted into a six-membered ring by way of the Diels-Alder reaction. ${ }^{37}$ At this time, according to this pathway, oxygen could interact with the $\alpha$-C of the ether bond to form two free radicals, ${ }^{36}$ which would have facilitated the aromatization of carbon atoms.

\section{Conclusions}

Using an oxygen atmosphere in the synthesis of CQDs from PEG 400 can dramatically enhance both the PL intensity and PLQY of the CQDs, and prolong their average lifetime at the same time. The mechanism of formation of these CQDs was indicated to involve PEG successively undergoing dehydration, dehydrogenation, polymerization and aromatization reactions, leading to the formation of the CQDs. Here, including oxygen in the dehydrogenation reaction can increase the degree of conjugation of the formed CQDs and increase the strength of the PL intensity.

\section{Acknowledgements}

We are grateful for the financial support of the National Natural Science Foundation of China (Grant No. 31470600) and Topnotch Academic Programs Project of Jiangsu Higher Education Institutions (PPZY2015C221).

\section{Notes and references}

1 X. Xu, R. Ray, Y. Gu, H. J. Ploehn, L. Gearheart, K. Raker and W. A. Scrivens, J. Am. Chem. Soc., 2004, 126, 12736-12737.

2 A. Cayuela, M. L. Soriano and M. Valcárcel, Anal. Chim. Acta, 2013, 804C, 246-251.

3 F. Yan, Y. Zou, M. Wang, X. Mu, N. Yang and L. Chen, Sens. Actuators, B, 2014, 192, 488-495.

4 L. Q. Liu, Y. F. Li, L. Zhan, Y. Liu and C. Z. Huang, Sci. China: Chem., 2011, 54, 1342-1347.

5 M. Zhou, Z. Zhou, A. Gong, Y. Zhang and Q. Li, Talanta, 2015, 143, 107-113.

6 S. Chandra, P. Das, S. Bag, D. Laha and P. Pramanik, Nanoscale, 2011, 3, 1533-1540.
7 W. Zonghua, X. Jianfei, Z. Chengfeng, V. Brian, X. Yanzhi, Z. Feifei, L. Yanhui, X. Linhua and T. Jie, Colloids Surf., B, 2013, 112C, 192-196.

8 S. Zhuo, M. Shao and S. T. Lee, ACS Nano, 2016, 6, 10591064.

9 Y. P. Sun, B. Zhou, Y. Lin, W. Wang, K. A. S. Fernando, P. Pathak, M. J. Meziani, B. A. Harruff, X. Wang and H. Wang, J. Am. Chem. Soc., 2006, 128, 7756-7757.

10 S. L. Hu, K. Y. Niu, J. Sun, J. Yang, N. Q. Zhao and X. W. Du, J. Mater. Chem., 2009, 19, 484-488.

11 J. Wang, C. F. Wang and S. Chen, Angew. Chem., 2012, 51, 9297-9301.

12 Q. L. Zhao, Z. L. Zhang, B. H. Huang, J. Peng, M. Zhang and D. W. Pang, Chem. Commun., 2008, 41, 5116-5118.

13 H. Liu, T. Ye and C. Mao, Angew. Chem., Int. Ed., 2007, 46, 6473-6475.

14 L. Tian, D. Ghosh, W. Chen, S. Pradhan, X. Chang and S. Chen, Chem. Mater., 2009, 21, 2803-2809.

15 M. J. Krysmann, A. Kelarakis and E. P. Giannelis, Green Chem., 2012, 14, 3141-3145.

16 A. Rahy, C. Zhou, J. Zheng, S. Y. Park, M. J. Kim, I. Jang, S. J. Cho and D. J. Yang, Carbon, 2012, 50, 1298-1302.

17 B. Zhang, C. Y. Liu and Y. Liu, Ber. Dtsch. Chem. Ges., 2010, 2010, 4411-4414.

18 Z. Ma, H. Ming, H. Huang, Y. Liu and Z. Kang, New J. Chem., 2012, 36, 861-864.

19 C. Fowley, B. Mccaughan, A. Devlin, I. Yildiz, F. M. Raymo and J. F. Callan, Chem. Commun., 2012, 48, 93619363.

20 F. M. Veronese and G. Pasut, Drug Discovery Today, 2005, 10, 1451-1458.

21 A. S. Karakoti, S. Das, S. Thevuthasan and S. Seal, Angew. Chem., 2011, 50, 1980-1994.

22 V. Milosavljevic, H. V. Nguyen, P. Michalek, A. Moulick, P. Kopel, R. Kizek and V. Adam, Chem. Pap., 2014, 69, 192201.

23 R. Liu, D. Wu, S. Liu, K. Koynov, W. Knoll and Q. Li, Angew. Chem., Int. Ed., 2009, 48, 4598-4601.

24 R. J. Fan, Q. Sun, L. Zhang, Y. Zhang and A. H. Lu, Carbon, 2014, 71, 87-93.

25 M. Chen, W. Wang and X. Wu, J. Mater. Chem. B, 2014, 2, 3937-3945.

26 V. B. Kumar, Z. Porat and A. Gedanken, Ultrason. Sonochem., 2016, 28, 367-375.

27 K. Mackenzie, O. Dunens and A. T. Harris, Sep. Purif. Technol., 2009, 66, 209-222.

28 H. Zhu, X. Wang, Y. Li, Z. Wang, F. Yang and X. Yang, Chem. Commun., 2009, 34, 5118-5120.

29 A. Jaiswal, S. S. Ghosh and A. Chattopadhyay, Chem. Commun., 2012, 48, 407-409.

30 X. Sui and Z. Wang, Polym. Adv. Technol., 2013, 24, 593599.

31 S. Yang, J. Sun, C. Zhu, P. He, Z. Peng and G. Ding, Analyst, 2015, 141, 1052-1059.

32 D. Pan, J. Zhang, Z. Li, C. Wu, X. Yan and M. Wu, Chem. Commun., 2010, 46, 3681-3683.

33 B. De and N. Karak, RSC Adv., 2013, 3, 8286-8290. 
34 S. Zhu, J. Zhang, L. Wang, Y. Song, G. Zhang, H. Wang and B. Yang, Chem. Commun., 2012, 48, 10889-10891.

35 Z. C. Yang, X. Li and J. Wang, Carbon, 2011, 49, 5207-5212. 36 Z. Diao, B. Li, B. Yu, A. Liu and L. He, J. Energy Chem., 2013, 22, 363-367.
37 B. M. Jacobson, G. M. Arvanitis, C. A. Eliasen and R. Mitelman, J. Org. Chem., 1985, 50, 194-201.

38 J. N. Hemenway, T. C. Carvalho, V. M. Rao, Y. Wu, J. K. Levons, A. S. Narang, S. R. Paruchuri, H. J. Stamato and S. A. Varia, J. Pharm. Sci., 2012, 101, 3305-3318. 